\title{
Fluorescence diagnosis of lymph node metastasis of lung cancer in a mouse model
}

\author{
HIROMITSU TAKIZAWA ${ }^{1}$, KAZUYA KONDO ${ }^{2}$, HIROAKI TOBA ${ }^{1}$, \\ KOICHIRO KENZAKI ${ }^{1}$, SHOJI SAKIYAMA ${ }^{1}$ and AKIRA TANGOKU ${ }^{1}$

\begin{abstract}
Departments of ${ }^{1}$ Thoracic, Endocrine Surgery and Oncology, and ${ }^{2}$ Oncological Medical Services, Institute of Health Bioscience, The University of Tokushima Graduate School, 3-18-15, Kuramotocho, Tokushima 770-8503, Japan
\end{abstract}

Received February 18, 2009; Accepted March 20, 2009

DOI: $10.3892 /$ or_00000400

\begin{abstract}
Fluorescence diagnosis of lymph node metastasis of non-small cell lung cancer has not been reported previously. This study was conducted to evaluate the feasibility of fluorescence detection using 5-aminolevulinic acid and mono-Laspartyl chlorine e6 for lymph node metastasis in a lung cancer mouse model. Human lung cancer cell line Ma44-3, which forms metastatic lymph nodes in the mediastinum, was injected into the left lungs of 6 severe combined immunodeficiency disease mice. Two weeks after implantation, 2 groups of 3 mice received oral administration of 5-aminolevulinic acid $(100 \mathrm{mg} / \mathrm{kg})$ or intraperitoneal administration of mono-L-aspartyl chlorine e6 $(5 \mathrm{mg} / \mathrm{kg})$. Both lungs and mediastinal organs were removed en-bloc and illuminated with blue light $(405 \mathrm{~nm})$ to evaluate the detectability of lung tumors and metastatic lymph nodes in the mediastinum. These organs were evaluated histopathologically. Clear red fluorescence was observed in the lung tumors of all mice. Metastatic lymph nodes had formed in 5 of 6 mice and were detected by fluorescent detection in all 5 mice even though one of the lymph nodes was invisible macroscopically under white light. In conclusion, fluorescence diagnosis of lymph node metastasis is possible in a mouse model. The successful result with small lymph node metastasis suggests the possibility of clinical application.
\end{abstract}

\section{Introduction}

Information on lymph node metastasis is essential for optimizing treatment strategies for non-small cell lung cancer (NSCLC) $(1,2)$; however, the methodology of clinical and surgical nodal staging of NSCLC remains controversial.

Correspondence to: Dr Kazuya Kondo, Department of Oncological Medical Services, Institute of Health Bioscience, The University of Tokushima Graduate School, 3-18-15, Kuramotocho, Tokushima 770-8503, Japan

E-mail: kondo@clin.med.tokushima-u.ac.jp

Key words: lung cancer, lymph nodes
Clinical nodal staging is determined by combining various radiologic examinations with invasive histopathologic examinations. Positron emission tomography and computed tomography using fluorodeoxyglucose (FDG PET-CT) has been rapidly accepted as the standard non-invasive modality for staging in patients with NSCLC. FDG PET-CT is superior to CT, especially in nodal staging of NSCLC (3). Despite improved accuracy, positive nodes detected by FDG PET-CT require histopathologic confirmation because of the high false-positive rate from coexisting inflammatory or infectious processes (4). Cervical mediastinoscopy or endobronchial ultrasound-guided transbronchial needle aspiration is usually performed in patients with suspected N2 disease, and cN0-1 (c-stage I-II) patients proceed to surgical resection without preoperative therapy. In surgical treatment, systematic mediastinal lymph node sampling or dissection is recommended for accurate staging (5), which crucially means that existing modalities for clinical nodal staging can not replace surgical staging. However, there is a dilemma that if a surgeon explores more lymph nodes intraoperatively for accurate staging, unexpected complications increase, therefore, we conducted a study of fluorescence diagnosis with a photosensitizer of lymph node metastasis of NSCLC, which would aid intraoperative nodal staging. Recent studies support segmentectomy for peripheral stage IA NSCLC of $2 \mathrm{~cm}$ or less $(6,7)$. Because intraoperative confirmation of pathological $\mathrm{N} 0$ is essential for patients who proceed to radical segmentectomy, intraoperative nodal staging becomes important.

5-Aminoleavulinic acid (ALA) is a natural precursor in the heme pathway. It is difficult for administered ALA to reach the last step of the pathway in tumor cells because it lacks a negative feedback mechanism and has reduced enzymatic activity. This leads to the accumulation of protoporphyrin IX (PpIX), a metabolite of ALA. PpIX becomes a fluorescent agent when stimulated by light of a specific wavelength. Mono-L-aspartyl chlorine e6 (talaporfin sodium, laserphyrin, NPe6), a second-generation photosensitizer, accumulates in tumor cells and becomes a phototoxic agent in photodynamic therapy. Both photosensitizers have almost the same excitation light wavelength of around $405 \mathrm{~nm}$. Under excitation with this blue light, these photosensitizers emit red fluorescence. With this mechanism, fluorescence detection using ALA for bladder cancer (8) or NPe6 for centrally located early lung cancer (9) have been applied clinically; however, there are 
only a few reports on fluorescence diagnosis using a photosensitizer for lymph node metastasis of cancers (10-12).

We established an orthotopic implantation severe combined immunodeficiency disease (SCID) mouse model of human lung cancer (13). With this model, human lung cancer cell line Ma44-3 injected into the left lung of the mouse forms a lung tumor and metastasizes to mediastinal lymph nodes, which is considered to closely resemble the clinical lymphogenous spread of lung cancer. This study was conducted to evaluate the feasibility of fluorescence detection using ALA and NPe6 for lymph node metastasis in a mouse model.

\section{Materials and methods}

This study was approved by The Committee for the Care and Use of Animals in The University of Tokushima Faculty of Medicine and was performed by the Institutional Animal Care and Oversight Committee according to established guidelines.

Animals. Male SCID mice (6 weeks of age) with a CB-17 genetic background were purchased from CLEA Japan, Inc. (Tokyo, Japan). These mice had been raised from birth in a specific pathogen-free environment.

Cell line. Ma44-3, which was cloned from Ma44 (human squamous cell lung cancer cell line) in our laboratory, was cultured in RPMI-1640 with 10\% heat-inactivated fetal bovine serum (Bioscience Pty, Ltd., Australia) and maintained at $37^{\circ} \mathrm{C}$ in a humidified incubator with $5 \% \mathrm{CO}_{2}$ in air.

Orthotopic intrapulmonary implantation procedure. As shown in our previous studies (13), Ma44-3 cells were harvested for implantation at 70-80\% confluence using $1 \mathrm{mmol} / \mathrm{l}$ EDTA (Wako Pure Chemical Industries, Ltd., Osaka, Japan) in PBS (Nissui Pharmaceutical Co., Ltd., Tokyo, Japan). The cells were washed in RPMI-1640 and resuspended to a final concentration of $2.0 \times 10^{6}$ cells $/ \mathrm{ml}$ in RPMI-1640 containing $0.1 \%$ BSA (Boehringer Mannheim, Mannheim, Germany). The mice were fully anesthetized by ether inhalation. The experimental mice were placed in the right lateral decubitus position with all four limbs restrained. A $1-\mathrm{cm}$ transverse incision was made in the left lateral skin just below the inferior border of the scapula of the SCID mouse. The muscles were separated from the ribs by sharp dissection, and the intercostal muscles were exposed, revealing the left lung. A 30 -gauge needle was inserted $5 \mathrm{~mm}$ into the lung through the intercostal muscle, and an inoculum of $2.0 \times 10^{6}$ tumor cells/ml with $400 \mu \mathrm{g} / \mathrm{ml}$ Matrigel (Collaborative Biomedical Products, Bedford, MA) was then injected into the left lung in a final volume of $10 \mu 1\left(2.0 \times 10^{4}\right.$ cells $)$ of medium. The skin incision was closed with 3-0 silk. Based on our previous experiments, tumor growth was allowed for 2 weeks. In our previous experiment, after Ma44-3 cells were orthotopically implanted into SCID mice, a macroscopically detectable tumor was formed at the site of injection on day 9 , and mediastinal lymph node metastasis was observed on day 12 (13).

Photosensitization. Two groups of 3 mice received an oral administration of ALA $(100 \mathrm{mg} / \mathrm{kg})$ or intraperitoneal adminis- tration of NPe6 (5 mg/kg) 3-4 h before fluorescence diagnosis. The mice were then kept under subdued light conditions to avoid photobleaching and phototoxic reactions.

Fluorescence diagnosis. The SCID mice were sacrificed and both lungs and mediastinal organs were removed en-bloc. These organs were immediately observed under white light and then illuminated by blue light (405 nm) using VLD-M1 (M\&M Co., Ltd., Tokyo, Japan) to evaluate the detectability of lung tumors and metastatic lymph nodes in the mediastinum. All tissues suspected of lymph node metastasis by fluorescence diagnosis were sampled and stored separately.

Histology. All organs and tissues were investigated microscopically after staining with hematoxylin and eosin.

\section{Results}

Lung tumors, 3-5 $\mathrm{mm}$ in size, were identified in the left lung of all 6 mice under white light. All lung tumors emitted clear red fluorescence under fluorescence detection and were comfirmed histopathologically (Fig. 1A-C). Histopathologic exploration revealed metastatic mediastinal lymph nodes in 2 of 3 mice administered ALA and 3 of 3 mice administered NPe6 (Table I). Under white light, lymphadenopathy suggesting metastasis was recognized in 2 of 3 mice administered ALA and 2 of 3 mice administered NPe6. Under fluorescence detection, these lymph nodes presenting with lymphadenopathy emitted clear red fluorescence. In one mouse administered NPe6, despite no lymphadenopathy, small but clear red fluorescence was observed in the mediastinal tissue, which was proved to be a metastatic lymph node histopathologically (Fig. 1D-F).

\section{Discussion}

Information on lymph node metastasis is of paramount importance in optimizing treatment strategies for NSCLC. A recent trial of adjuvant chemotherapy that randomized 840 completely resected patients with stages I to IIIA to four postoperative cycles of cisplatin and navelbine vs. observation demonstrated the long-term 5-year survival of stage IIIA patients in the chemotherapy arm (42 vs. 26\%) (1). The benefit of adjuvant chemotherapy was also seen in stage II patients but not in stage I. In ACCP Evidence-Based Clinical Practice Guidelines (2nd edition), platinum-based chemotherapy is recommended for patients with resected NSCLC who were found to have incidental (occult) N2 disease (2).

The optimal surgical exploration to deal with mediastinal lymph nodes remains controversial. Two randomized trials demonstrated no difference in overall survival between the lymph node dissection group and lymph node sampling group $(14,15)$. In contrast, another trial showed improved survival for patients with clinical stage I-III NSCLC who had undergone lymph node dissection compared with sampling (16). From a recent ongoing trial by ACOSOG, which was designed to compare survival between the lymph node dissection group and sampling group, preliminary data have been reported. No difference in operative mortality was found between the two groups; however, lymph node dissection was associated with 

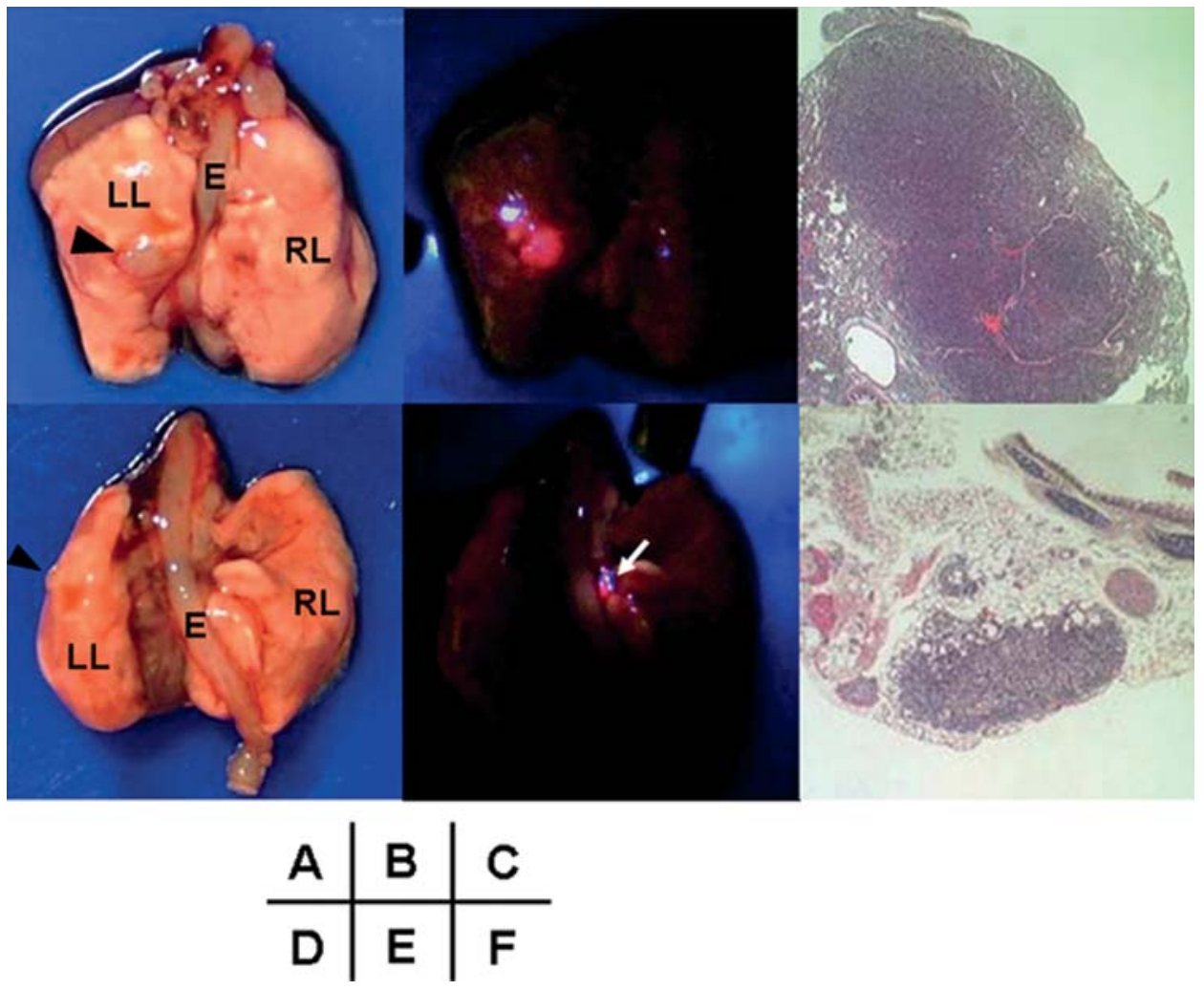

Figure 1. (A) Specimen of a mouse administered 5-aminoleavulinic acid. Arrow head, tumor; LL, left lung; RR, right lung; E, esophagus. (B) Fluorescence diagnosis of the same specimen shown in (A). Red fluorescence at the tumor. (C) Histopathologic findings of the tumor in (B). (D) Specimen of a mouse administered talaporfin sodium. No lymphadenopathy suggesting metastasis is seen in the mediastinum. Arrow head, tumor; LL, left lung; RR, right lung; E, esophagus. (E) Fluorescence diagnosis of the same specimen shown in (D). Small red fluorescence is seen in the mediastinum (arrow). (F) Histopathologic finding of the tissue in (E) showing a metastatic lymph node.

Table I. Detectability of lymph node metastasis.

\begin{tabular}{ccccc}
\hline Mouse & Photosensitizer & LN metastasis & WL & FD \\
\hline 1 & ALA & + & + & + \\
2 & ALA & + & + & + \\
3 & ALA & - & - & - \\
4 & NPe6 & + & - & + \\
5 & NPe6 & + & + & + \\
6 & NPe6 & + & + & + \\
\hline
\end{tabular}

ALA, 5-aminoleavulinic acid; NPe6, mono-L-aspartyl chlorine e6; LN metastasis, lymph node metastasis; WL, white light; FD, fluorescence diagnosis.

longer operative time and a greater volume of postoperative chest tube drainage (17). Several authors have described increased complications or surgical stress concerning lymph node dissection $(18,19)$. To reduce the extent of lymph node exploration, studies of lobe-specific lymph node dissection and sentinel lymph node sampling have been performed (19-21). If intraoperative fluorescence diagnosis for lymph node infiltration becomes clinically feasible, less invasive selective lymph node sampling will be supported.

Intraoperative nodal staging is also essential for patients with small early-stage NCSLC. Increased opportunity for CT scans is identifying more patients with a small peripheral lung lesion. Because of such a background, the strategy for the treatment of patients with small early-stage NCSLC has been changing. Recent studies have suggested that segmentectomy can be an alternative to lobectomy in the treatment of patients with peripheral stage IA NSCLC of $2 \mathrm{~cm}$ or less $(6,7)$. These studies showed no inferior recurrence rate or prognosis of patients who had received a segementectomy compared with patients who had received a lobectomy. These results are based on the fact that only a few patients are pathologically N1 or N2 and margin-positive after segmentectomy in patients with peripheral stage IA NSCLC of $2 \mathrm{~cm}$ or less. In these studies, the authors described that intraoperative sampling of lobar, hilar and mediastinal lymph node had been performed to confirm N0; however, sampling of all related lymph nodes and checking them by frozensection examination in every patient who undergoes a segmentectomy requires an enormous effort by both surgeons and pathologists. Patients who receive inadequate exploration of lymph nodes may have a disadvantage from less resection; therefore, intraoperative nodal staging is essential for patients with small early-stage NCSLC who proceed to radical segmentectomy. Fluorescence detection of infiltrated lymph nodes may become a supplementary diagnostic tool at the time of determining segmentectomy.

Nomori et al investigated the utility of sentinel node navigation segmentectomy for clinical stage IA NSCLC (22). Their procedure identified sentinel lymph nodes in 43 (83\%) 
patients, and 3 patients were proved to have metastatic sentinel nodes, and were converted to a lobectomy or larger segmentectomy. The authors insisted on the importance of accurate intraoperative nodal staging in sublober resection for earlystage NSCLC patients. Sugi et al showed the possibility of sentinel lymph node sampling and also described the importance of accurate lymph node staging in patients with earlystage NSCLC (21). As shown in our study, fluorescence diagnosis is useful to detect nodal infiltration; however, the concept is different from sentinel node detection. The sentinel concept is to identify the first lymph nodes draining a solid and malignant tumor. Fluorescence diagnosis with a photosensitizer for lymph nodes aims to identify metastatic infiltrated lymph nodes.

In the present study, fluorescence detection using a photosensitizer was possible for all mice with infiltrated lymph nodes. Although the infiltrated lymph nodes were 1$2 \mathrm{~mm}$, clear red fluorescence was observed. Especially in one mouse administered NPe6, despite no macroscopically visible lymph node, small but clear red fluorescence was observed in a metastatic lymph node proved pathologically. These results suggest the high sensitivity of this method. Prosst et al reported the utility of fluorescence diagnosis using ALA for pleural malignancies in their nude rat model (23). In the study, fluorescence diagnosis using ALA detected more pleural disseminated lesions of lung cancer which were invisible under white light. There are several reports of fluorescence diagnosis for lymph node metastasis. A small clinical study suggested the possibility of fluorescence diagnosis using ALA for lymph node metastasis in patients with penile cancer (10). A case report of ovarian cancer demonstrated that metastatic infiltrated lymph nodes were identified by fluorescence diagnosis using ALA (11). A study of breast cancer showed higher fluorescence intensity in metastatic lymph nodes than in non-metastatic lymph nodes after application of ALA (12).

ALA and NPe6 have already been used clinically. ALA application has relatively few side effects, and allows good discrimination between malignant and non-malignant tissue (24). Fluorescence detection with ALA application has been widely used in urology. ALA-induced fluorescence cystoscopy improved the detectability of superficial bladder cancer and its recurrence-free survival (8). NPe6 was approved for use in the diagnosis and treatment of centrally located early lung cancer (9). A phase II clinical study of photodynamic therapy using NPe6 for early-stage lung cancer demonstrated excellent anticancer effects and safety, including reduced skin phototoxicity (25).

In conclusion, fluorescence diagnosis of lymph node metastasis is possible in a mouse model. The successful result with small lymph node metastasis suggests the possibility of clinical application. Further investigations are expected for clinical use.

\section{References}

1. Douillard JY, Rosell R, De Lena M, et al: Adjuvant vinorelbine plus cisplatin versus observation in patients with completely resected stage IB-IIIA non-small cell lung cancer [Adjuvant Navelbine International Trialist Association (ANITA)]: a randomised controlled trial. Lancet Oncol 7: 719-727, 2006.
2. Robinson LA, Ruckdeschel JC, Wagner H Jr and Stevens CW; American College of Chest Physicians: Treatment of non-small cell lung cancer-stage IIIA: ACCP evidence-based clinical practice guidelines (2nd edition). Chest 132: 243-265, 2007.

3. Shim SS, Lee KS, Kim BT, et al: Non-small cell lung cancer: prospective comparison of integrated FDG PET/CT and CT alone for preoperative staging. Radiology 236: 1011-1019, 2005.

4. Lee BE, von Haag D, Lown T, Lau D, Calhoun R and Follette D: Advances in positron emission tomography technology have increased the need for surgical staging in non-small cell lung cancer. J Thorac Cardiovasc Surg 133: 746-752, 2007.

5. Scott WJ, Howington J, Feigenberg S, Movsas B and Pisters K; American College of Chest Physicians: Treatment of non-small cell lung cancer stage I and stage II: ACCP evidence-based clinical practice guidelines (2nd edition). Chest 132: 234-242, 2007.

6. Okada M, Koike T, Higashiyama M, Yamato Y, Kodama K and Tsubota N: Radical sublobar resection for small-sized non-small cell lung cancer: a multicenter study. J Thorac Cardiovasc Surg 132: 769-775, 2006.

7. Okumura M, Goto M, Ideguchi K, et al: Factors associated with outcome of segmentectomy for non-small cell lung cancer: long-term follow-up study at a single institution in Japan. Lung Cancer 58: 231-237, 2007.

8. Denzinger S, Burger M, Walter B, et al: Clinically relevant reduction in risk of recurrence of superficial bladder cancer using 5-aminolevulinic acid-induced fluorescence diagnosis: 8year results of prospective randomized study. Urology 69: 675-679, 2007.

9. Usuda J, Tsutsui H, Honda H, et al: Photodynamic therapy for lung cancers based on novel photodynamic diagnosis using talaporfin sodium (NPe6) and autofluorescence bronchoscopy. Lung Cancer 58: 317-323, 2007.

10. Frimberger D, Linke R, Meissner H, et al: Fluorescence diagnosis: a novel method to guide radical inguinal lymph node dissection in penile cancer. World J Urol 22: 150-154, 2004.

11. Hillemanns P, Reiff J, Stepp H and Soergel P: Lymph node metastasis detection of ovarian cancer by porphyrin fluorescence photodetection: case report. Lasers Med Sci 22: 131-135, 2007.

12. Frei KA, Bonel HM, Frick H, Walt H and Steiner RA: Photodynamic detection of diseased axillary sentinel lymph node after oral application of aminolevulinic acid in patients with breast cancer. Br J Cancer 90: 805-809, 2004.

13. Ishikura H, Kondo K, Miyoshi T, Kinoshita H, Hirose $T$ and Monden Y: Artificial lymphogenous metastatic model using orthotopic implantation of human lung cancer. Ann Thorac Surg 69: 1691-1695, 2000.

14. Izbicki JR, Passlick B, Pantel K, et al: Effectiveness of radical systematic mediastinal lymphadenectomy in patients with resectable non-small cell lung cancer: results of a prospective randomized trial. Ann Surg 227: 138-144, 1998.

15. Sugi K, Nawata K, Fujita N, et al: Systematic lymph node dissection for clinically diagnosed peripheral non-small cell lung cancer less than $2 \mathrm{~cm}$ in diameter. World J Surg 22: 290-294, 1998.

16. Wu Y, Huang ZF, Wang SY, Yang XN and Ou W: A randomized trial of systematic nodal dissection in resectable non-small cell lung cancer. Lung Cancer 36: 1-6, 2002.

17. Allen MS, Darling GE, Pechet TT, et al; ACOSOG Z0030 Study Group: Morbidity and mortality of major pulmonary resections in patients with early-stage lung cancer: initial results of the randomized, prospective ACOSOG Z0030 trial. Ann Thorac Surg 81: 1013-1019, 2006.

18. Bollen EC, van Duin CJ, Theunissen PH, vt Hof-Grootenboer BE and Blijham GH: Mediastinal lymph node dissection in resected lung cancer: morbidity and accuracy of staging. Ann Thorac Surg 55: 961-966, 1993.

19. Muraoka M, Akamine S, Oka T, et al: Sentinel node sampling limits lymphadenectomy in stage I non-small cell lung cancer. Eur J Cardiothorac Surg 32: 356-361, 2007.

20. Asamura H, Nakayama H, Kondo H, Tsuchiya $R$ and Naruke T: Lobe-specific extent of systematic lymph node dissection for non-small cell lung carcinomas according to a retrospective study of metastasis and prognosis. J Thorac Cardiovasc Surg 117: 1102-1111, 1999

21. Sugi K, Kaneda Y, Sudoh M, Sakano H and Hamano K: Effect of radioisotope sentinel node mapping in patients with cT1 N0 M0 lung cancer. J Thorac Cardiovasc Surg 126: 568-573, 2003. 
22. Nomori H, Ikeda K, Mori T, et al: Sentinel node navigation segmentectomy for clinical stage IA non-small cell lung cancer. J Thorac Cardiovasc Surg 133: 780-785, 2007.

23. Prosst RL, Winkler S, Boehm E and Gahlen J: Thoracoscopic fluorescence diagnosis (TFD) of pleural malignancies: experimental studies. Thorax 57: 1005-1009, 2002.
24. Peng Q, Warloe T, Berg K, et al: 5-Aminolevulinic acid-based photodynamic therapy. Clinical research and future challenges. Cancer 79: 2282-2308, 1997.

25. Kato H, Furukawa K, Sato M, et al: Phase II clinical study of photodynamic therapy using mono-L-aspartyl chlorin e6 and diode laser for early superficial squamous cell carcinoma of the lung. Lung Cancer 42: 103-111, 2003. 